\title{
LA INVESTIGACIÓN FORMATIVA A TRAVÉS DEL APRENDIZAJE ORIENTADO A PROYECTOS: UNA PROPUESTA DE INNOVACIÓN EN EL GRADO DE PEDAGOGÍA
}

\author{
FORMATIVE RESEARCH THROUGH PROJECT-ORIENTED LEARNING: \\ AN INNOVATION IN PEDAGOGY DEGREE
}

\author{
Ruth Vilà Baños \\ ruth_vila@ub.edu \\ M. José Rubio Hurtado \\ mjrubio@ub.edu \\ Vanesa Berlanga Silvente \\ berlanga.silvente@ub.edu \\ Universidad de Barcelona
}

\section{RESUMEN}

La investigación formativa sitúa al alumnado como protagonista activo de su propio proceso de aprendizaje. El rol del profesorado es acompañar a los estudiantes hacia el aprendizaje independiente, motivándolos a trabajar de forma autónoma. El alumnado adopta un rol activo, trabaja en equipo, busca información, y planifica el trabajo, tomando sus propias decisiones, integrando conocimientos, y desarrollando competencias diversas. En esta innovación apostamos por el aprendizaje orientado a proyectos (ABP) y la evaluación con portafolio digital, como estrategias para la investigación formativa. La finalidad del estudio que presentamos es el análisis de la innovación en una asignatura del grado de Pedagogía, desde la perspectiva del alumnado participante; mediante el método por encuesta, orientada al estudio de caso. Los principales resultados obtenidos evidencian la idoneidad de la innovación docente implementada para potenciar el desarrollo de habilidades de pensamiento de orden superior. El $\mathrm{ABP}$ favorece la adquisición e integración de los nuevos conocimientos, destacamos especialmente, el desarrollo de dos competencias básicas como son el trabajo colaborativo y la capacidad de reflexión. Para el desarrollo de estas competencias, han resultado especialmente útiles, según el alumnado, las actividades de aprendizaje autónomo, las actividades colaborativas, las del proyecto de investigación y el portafolio. El aspecto con el que el alumnado se siente más satisfecho es el nivel de autonomía alcanzado tras cursar la asignatura. Este resultado evidencia los beneficios de la metodología docente no directiva, donde el alumnado adopta un rol activo y el profesorado ofrece feed back, guía el proceso de aprendizaje y evalúa. Por todo concluimos que alentar el aprendizaje a través de proyectos está demostrando efectos positivos en el fomento de la investigación, en la medida que el alumnado se involucra en un proceso dinámico e interactivo de aprendizaje.

Palabras clave: investigación formativa, aprendizaje orientado a proyectos, portafolio digital, innovación docente. 


\begin{abstract}
Formative research puts the students as active creators of their own learning process. The role of the teacher is to accompany students towards independent learning, motivating them to work independently. The students take an active role, working in teams, looking for information, and plan the work, taking their own decisions, integrating knowledge, and developing various competences. We propose an innovation based on project- oriented learning (PBL) and digital portfolio assessment as formative research strategies for learning. The purpose of the present study is the analysis of innovation in a course grade of Pedagogy, from the perspective of participating students; using the survey method, and case study. The main results obtained show the adequacy of the teaching innovation implemented to enhance the development of skills of higher order thinking. PBL promotes the acquisition and integration of new knowledge, especially include the development of two basic competences such as collaborative work and thinking skills. To develop these competences, students have shown as particularly useful: independent learning activities, collaborative activities, research and project portfolio. The aspect that students feel more satisfied is the level of autonomy achieved after completing the course. This result shows the benefits of non-directive teaching methodology where students take an active role, and teachers provides feedback, guide the learning process and evaluate. For all we conclude that project-oriented learning is demonstrating positive effects on the promotion of research, to the extent that students are involved in a dynamic and interactive learning process.
\end{abstract}

Keywords: formative research, project-oriented learning, digital portfolio, teaching innovation.

\title{
1. LA INVESTIGACIÓN FORMATIVA
}

Una de las funciones de la Educación Superior es capacitar al alumnado para producir conocimientos, aplicar habilidades y seguir aprendiendo a lo largo de su carrera profesional. Pero para que una persona posea la capacidad de producir conocimientos y de aprendizaje permanente, se requiere otra capacidad, que según Miyahira (2009), es la capacidad de investigación, siendo la investigación una actividad orientada a la generación de conocimiento, contrastación de modelos teóricos con la realidad, planteamiento de nuevos problemas o resolución de problemas prácticos (Bunge, 1983; Arnau, 1996; Del Rincón et al, 1995). Estas acciones son de gran relevancia en nuestra sociedad, por lo que la universidad también posee la función de capacitar a los alumnos para investigar.

Guerrero (2007), propone el término de formación para la investigación, refiriéndose al conjunto de acciones orientadas a favorecer la apropiación y desarrollo de los conocimientos, habilidades y actitudes necesarias para que estudiantes y profesores puedan desempeñar con éxito actividades productivas asociadas a la investigación científica, el desarrollo tecnológico y la innovación, ya sea en el sector académico o en el profesional. En este sentido, la investigación formativa se presenta como una actividad con capacidad para fomentar las capacidades ligadas a la investigación, diferenciándose de la formación en investigación en el enfoque: en un caso se trata de un enfoque curricular y en el otro de un enfoque de metodología docente. La investigación formativa se sitúa en este último, pues como señala Guerrero (2007), contempla la investigación como herramienta del proceso enseñanza-aprendizaje, siendo su finalidad difundir información existente y favorecer que el estudiante la incorpore como conocimiento (aprendizaje). Dicho conocimiento puede versar en cualquier área o especialidad, siendo la investigación un puro medio para desarrollar tal conocimiento. 
En la misma línea, Cortés-Nieto, et. al. (2008), abordan la relación docencia-investigación y nos sitúan en el marco de las posibles estrategias de enseñanza-aprendizaje a utilizar en la educación superior centradas en el alumnado y con efectividad demostrada para el desarrollo competencial, en coherencia con el marco que nos ofrece el Espacio Europeo de Educación Superior. Tal como plantea Pérez-Pérez (2013), el docente pasa a ser guía o catalizador del proceso de enseñanzaaprendizaje, promoviendo un rol activo en el estudiante, y proponiendo metodologías orientadas al aprendizaje activo, autónomo y participativo.

La investigación formativa, igualmente denominada "la enseñanza a través de la investigación" o "la docencia investigadora" (Parra, 2004), se entiende como una modalidad de investigación que favorece el paradigma metodológico actual que ha propiciado el proceso de convergencia europea, en la medida que sitúa al alumnado como protagonista activo de su propio proceso de aprendizaje basado en competencias (Villa y Poblete, 2007), dentro de un marco curricular formalmente definido. Según Parra (2004) la investigación formativa tiene dos características adicionales fundamentales: es una investigación orientada por un profesor, como parte de su función docente y los agentes investigadores no son profesionales de la investigación, sino alumnos en formación.

Según Sabariego (2012) la investigación formativa tiene dos acepciones que la definen y configuran: como un medio de formación en y para la investigación, y como una herramienta para la transformación en la acción o la práctica docente. En este sentido, la investigación formativa se centra en tres grandes principios (Sabariego, Ruiz y Sánchez 2013):

- La pregunta "la duda": el aprendizaje es el resultado de procesos de construcción del conocimiento por parte del alumnado, asumiendo un rol activo de autoaprendizaje y autogestor del mismo.

- La no directividad docente: la búsqueda formativa requiere una forma de relacionarse con los estudiantes como facilitador del aprendizaje, como orientador y guía experto, respetando los diferentes puntos de vista que surgen del trabajo. De esta manera, se favorece el aprendizaje autónomo.

- La docencia inductiva, con interacción entre el entorno, la comunidad educativa y el currículo. El concepto mismo de problema de investigación, entendido como un núcleo temático complejo de indagación necesariamente articulado con otros, permite múltiples aproximaciones (interdisciplinariedad), el diálogo de saberes sobre el objeto de enseñanza para su comprensión integral.

Estos principios promueven unos roles en el profesorado y alumnado determinados coherentes con la investigación formativa, así como unos métodos y estrategias favorecedores y potenciadores del autoaprendizaje, la autonomía y la interdisciplinariedad, que abordamos a continuación.

\section{LOS ROLES DEL PROFESORADOY ALUMNADO EN LA INVESTIGACIÓN FORMA- TIVA}

En la sociedad del conocimiento, la calidad de la educación superior está íntimamente asociada con la práctica de la investigación, práctica que se manifiesta de dos maneras: enseñar $a$ 
investigar y hacer investigación (Restrepo, 2003). Por tratarse de un problema pedagógico y didáctico es menester iniciar su estudio desde las estrategias de enseñanza, ya que su presencia es consustancial: la de aprendizaje por descubrimiento y construcción (Restrepo, 2003). La investigación formativa como transformación implica la estrategia del aprendizaje por descubrimiento y construcción (Elliot, 1994), donde el profesor plantea situaciones problemáticas, a veces ni siquiera problemas acabados o bien estructurados, dejando el trabajo de la solución al estudiante. En el aprendizaje por descubrimiento operan conjuntamente y colaborativamente tanto el estudiante como el profesor, más si se orienta por el método aprendizaje basado en problemas o per el método de aprendizaje basado en proyectos.

Desde esta perspectiva, el rol del profesorado se centra en la tutela a los estudiantes durante la elaboración del proyecto, ofreciéndoles recursos y orientación a lo largo de sus investigaciones. La ayuda se desplaza progresivamente del proceso al producto. Debe guiar a los estudiantes hacia el aprendizaje independiente, motivándolos a trabajar de forma autónoma, especialmente en las fases de planificación, realización y evaluación. Por su parte el alumnado adopta un rol activo, trabaja en equipo, busca información, planifica el trabajo, presenta informes y resultados al docente, tomando sus propias decisiones, integrando conocimientos, y desarrollando competencias diversas.

Cortés- Nieto et al. (2008) proponen diversas estrategias y métodos docentes para implementar la investigación formativa, entre las que destacan: el análisis de artículos de investigación, la vinculación de los estudiantes a proyectos de investigación en las asignaturas que estén cursando, o la técnica del portafolio.

\section{EL APRENDIZAJE ORIENTADO A PROYECTOS EN LA INVESTIGACIÓN FORMA- TIVA}

La innovación que supone la realización de proyectos como estrategia de aprendizaje radica no en el proyecto en sí mismo, sino en las posibilidades que supone su realización para poner en práctica y desarrollar diferentes competencias: el trabajo colaborativo, la capacidad para ver puntos de vista diferentes, la toma de decisiones, la capacidad para comunicar el proceso, entre otras (Moursund, 1999; Thomas, 2000; Tippelt y Lindemann, 2003).

La investigación formativa, en el terreno de la función pedagógica de la investigación, cuenta con métodos y prácticas de docencia investigativa que han demostrado cierta efectividad. Según De Miguel et al. (2006) los principales métodos de enseñanza pueden clasificarse en siete, tal como se resumen en la tabla 1.

En esta experiencia apostamos por método de aprendizaje orientado a proyectos (ABP) como punto de partida para la investigación formativa, ya que es de gran valor para vincular la educación superior a las necesidades de la sociedad (Soria, Sabariego y Donoso; 2013). El ABP es un un modelo de aprendizaje en el que los estudiantes planean, implementan y evalúan proyectos que tienen aplicación en el mundo real más allá del aula de clase (Blank, 1997; Harwell, 1997). Basado en el aprendizaje experiencial, tiene una gran importancia el proceso investigador alrededor de un tópico, con la finalidad de plantear soluciones a problemas complejos o abordar temas difíciles que 
permitan la generación de conocimiento nuevo y desarrollo de nuevas habilidades por parte de los estudiantes (Pérez Boullosa, 2006). Varios autores coinciden en afirmar que el método de proyectos reúne los requisitos necesarios para el desarrollo de competencias (Tippelt y Lindemann, 2003; Willard y Duffrin, 2003; Rodríguez-Sandoval et al., 2010).

\section{MÉTODO}

Método expositivo/Lección Magistral

Estudio de Casos

Resolución de ejercicios y problemas

Aprendizaje basado en problemas

Aprendizaje orientado a Proyectos

Aprendizaje cooperativo

Contrato de aprendizaje

\section{FINALIDAD}

Transmitir conocimientos y activar procesos cognitivos en el estudiante

Adquisición de aprendizajes mediante el análisis de casos reales o simulados

Ejercitar, ensayar y poner en práctica los conocimientos previos

Desarrollar aprendizajes activos a través de la resolución de problemas

Realización de un proyecto para la resolución de un problema, aplicando habilidades y conocimientos adquiridos

Desarrollar aprendizajes activos y significativos de forma cooperativa

Desarrollar el aprendizaje autónomo

Tabla 1. Métodos de enseñanza (adaptado de De Miguel, 2006)

Con este método pretendemos que los estudiantes asuman una mayor autonomía y responsabilidad de su propio aprendizaje, así como aplicar, en proyectos reales, las habilidades y conocimientos adquiridos en su formación. Es un aprendizaje orientado a la acción, en el que el profesor no constituye la fuente principal de acceso a la información. El propio estudiante es el que busca y selecciona las fuentes informativas, valora opciones y toma decisiones, tanto individual como cooperativamente. El trabajo en grupo es de gran relevancia en el ABP, en el que el producto final es fruto de las aportaciones integradas de cada uno de los miembros.

\section{EL PORTAFOLIO DIGITAL COMO ESTRATEGIA EVALUATIVA EN LA INVESTIGA- CIÓN FORMATIVA}

El portafolio supone una oportunidad de hacer investigación formativa en torno a la práctica evaluativa (Cortés-Nieto et al., 2008). Se orienta a la evaluación basada en desempeño y permite apreciar el desarrollo de múltiples habilidades en el estudiante, amén del saber particular que se quiere comprobar. La experiencia, para el docente y para el estudiante, demanda tareas similares a las desarrolladas en la práctica investigativa (Restrepo, 2003).

En consonancia con los objetivos del Espacio Educativo de Educación Superior, orientados a que las metodologías docentes enfaticen el proceso de aprendizaje y doten de un mayor protagonismo a los estudiantes, los portafolios se presentan como un recurso con un gran potencial para la 
formación integral del estudiante y para la evaluación formativa y la evaluación auténtica (Rodríguez Illera, 2009; Cambridge, 2010; Sabirón, 2013), orientada al trabajo y al ejercicio profesional y ofreciéndole al alumnado oportunidades para explicar, dialogar e investigar (Wiggins, 1990).

El portafolio, desde la perspectiva constructivista, favorece el desarrollo de procesos educativos desde narrativas intersubjetivas, centrados en la acción del estudiante consecuente con procesos de asimilación e interiorización. El estudiante auto-dirige su aprendizaje y aumenta su motivación intrínseca (Barrett, 2010).

Derivados del portafolio tradicional, los portafolios digitales suponen una alternativa de evaluación en la que por medio de un conjunto de recursos electrónicos profesorado y alumnado pueden almacenar, crear, seleccionar, organizar y editar los trabajos o evidencias de aprendizaje. La progresiva penetración de las TIC en la Educación Superior, está ayudando a la extensión de su uso a numerosas disciplinas.

El uso de estas herramientas tecnológicas genera una serie de ventajas para los usuarios, como el desarrollo de la habilidad en el uso del lenguaje, una mejor estructuración en el desarrollo de su trabajo, o una integración de los contenidos aprendidos, haciéndoles responsables de su aprendizaje, motivándoles y dando sentido a su trabajo (South, 2001).

Diversos autores también han valorado el portafolio digital como instrumento que ayuda al proceso de aprendizaje y al desarrollo de competencias transversales, como la planificación y organización, la toma de decisiones o la reflexión y autocrítica (Vilà, Rubio y Torrado, 2013; Rubio y Galván, 2013; Cambridge, 2010; Zubizarreta, 2009; Stefani, Mason, y Pegler, 2007).

Investigaciones recientes de portafolios digitales en Educación Superior, constatan las ventajas de estas herramientas para el desarrollo de competencias en los estudiantes. Aguaded et al. (2013), destacan, en su investigación centrada en el grado de Educación Social, que los portafolios digitales pueden favorecer una evaluación formativa y holística, al ser factible por parte de los docentes el seguimiento detallado sobre la evolución y las dificultades didácticas de los grupos de estudiantes. Y desde la óptica del alumnado, el portafolio ayuda a conocer el progreso del aprendizaje, ayuda a la planificación estructural de la materia objeto de estudio, estimulando la responsabilidad, la toma de decisiones y la resolución de conflictos (en la misma línea de los resultados encontrados por Todorova et al., 2010). Por su parte Sabiron y Arraiz (2012), concluyen en su experiencia de portafolios digitales en Psicopedagogía que estos promueven la transformación de los hábitos de estudio generando una mayor autonomía y dedicación continuada al aprendizaje, aunque resulta clave la tutorización: el portafolio provoca en sí mismo la necesidad de dialogar, consigo mismo, con los compañeros y los profesores: se trata de avanzar en el desarrollo de competencias a través de la acción-reflexión.

La reciente tesis doctoral de Castaño (2014), destaca la influencia que puede tener el portafolios digital en el aprendizaje de los estudiantes, aunque refiere también la importancia de ciertos factores para ese aprendizaje, con los que hay que contar: el enfoque de aprendizaje de los estudiantes (Biggs, 2001; Rodríguez Illera et al., 2013), las características de la evaluación, la alineación de la planificación didáctica con la propia herramienta de portafolios, así como las características de ésta, la formación inicial recibida en la herramienta, o la capacidad de reflexión. 


\section{CONTEXTO DE LA INNOVACIÓN}

En los títulos de Grado las oportunidades para hacer investigación formativa son numerosas: idear hipótesis, diseñar procesos, recopilar información, procesar datos, discutir, argumentar, interpretar, inferir y defender resultados son objetivos operativos clave para diseñar propuestas en este sentido.

La innovación se llevó a cabo en diferentes asignaturas de los grados de Pedagogía y Formación del profesorado de la Universidad de Barcelona (bajo el proyecto: El valor de la investigación formativa para la innovación y el desarrollo competencial en la educación superior, concedido en la Convocatoria de Ayudas del Programa de Mejora e Innovación Docente de la Universidad de Barcelona, IP: Marta Sabariego), aunque en este artículo se presenta sólo la experiencia de la asignatura Informática aplicada a la investigación educativa del grado de Pedagogía.

Se trata de una asignatura optativa de carácter metodológico de 3 créditos, que tiene por objetivo introducir al alumnado en el análisis de datos cuantitativos mediante programario informático. La asignatura forma parte del itinerario metodológico-investigativo optativo de la carrera tal como se recoge en la tabla 2 .

\begin{tabular}{|c|c|c|c|c|}
\hline $\begin{array}{c}\text { Tipo de } \\
\text { asignatura }\end{array}$ & 1er Curso & $2^{\circ}$ Curso & 3er Curso & $\begin{array}{c}4^{\circ} \\
\text { Curso }\end{array}$ \\
\hline Obligatorias & $\begin{array}{l}\text { Teoría y } \\
\text { práctica de la } \\
\text { investigación } \\
\text { educativa } \\
\text { (6 créditos })\end{array}$ & $\begin{array}{l}\text { - Estadística aplicada a la } \\
\text { educación } \\
\text { ( } 6 \text { créditos }) \\
\text { - Instrumentos y estrategias de } \\
\text { recogida de información ( } 6 \\
\text { créditos })\end{array}$ & $\begin{array}{l}\text { Diagnóstico } \\
\text { y orientación } \\
\text { educativa } \\
\text { ( } 6 \text { créditos) }\end{array}$ & \\
\hline Optativas & $\begin{array}{c}\text { No hay oferta } \\
\text { en el título }\end{array}$ & \multicolumn{3}{|c|}{$\begin{aligned} \text { - Resolución de problemas a través de investigación educativa (3 } \\
\text { créditos } 3 \text { er } \mathrm{S}) \\
\text { - Informática aplicada a la investigación educativa } \\
\\
\left.\quad \text { (3 créditos- } \mathbf{4}^{\circ} \mathbf{S}\right) \\
\text { - }\end{aligned}$} \\
\hline
\end{tabular}

Tabla 2. Distribución de las asignaturas de metodología de investigación en el título de Pedagogía de la Universidad de Barcelona (elaboración propia)

Las sesiones de clase se desarrollan íntegramente en un aula de informática y la baja ratio de alumnado permite la tutorización continua por parte del profesorado. Características que fueron consideradas favorables para el desarrollo de la investigación formativa a través del aprendizaje orientado a proyectos y del portafolio digital, método y estrategia idóneas para ser trabajadas desde esta perspectiva, por estar centradas en el estudiante.

La aplicación de esta innovación se llevó a cabo durante el curso 2012-2013, donde el profesorado asumió un rol no directivo, facilitador del aprendizaje, consistente en escasas clases magistrales y la tutorización en la misma aula de las actividades de aprendizaje. La evaluación consistió 
en la realización grupal de una investigación completa (sobre un tema libremente escogido por el alumnado), evidenciándola en un portafolio digital, el cual se constituyó como la herramienta para contener las fases de la investigación y otras evidencias de carácter libre. Cada alumno dispuso de un portafolio individual y otro grupal y los diseños se dejaron a la libre elección del alumnado. El portafolio grupal incluyó el proyecto de investigación y el individual otras actividades. Para cada evidencia de aprendizaje se diseñaron rúbricas de evaluación, y para el aprendizaje de la plataforma del portafolio digital (www.portafolis.ub.edu) se realizó una sesión formativa al alumnado.

El proceso seguido para implementar la innovación se inició con el consenso, por parte del equipo docente implicado, sobre las estrategias que el profesorado aplicaría en sus clases y sobre las responsabilidades que el alumnado debería asumir (tal y como se resumen en los cuadros 1 y 2). Seguidamente se aplicaron estas estrategias durante el curso y finalmente se evaluó los portafolios, el cual fue presentado en el aula por cada grupo.

El profesorado tutela a los estudiantes durante la elaboración del proyecto ofreciéndoles recursos y orientación a lo largo de su investigación. La ayuda se desplaza progresivamente del proceso al producto. Deberá estar disponible para aclarar las dudas del estudiante, así como guiarle hacia el aprendizaje independiente, motivándolo a trabajar de forma autónoma, especialmente en las fases de planificación, realización y evaluación.

Las tareas del profesor de forma secuenciada son:

- Presentación y definición del proyecto: estudiar un fenómeno de interés educativo libremente escogido por el alumnado aplicando y analizando un cuestionario también diseñado por aquél, que permita dar respuesta a los interrogantes planteados y generar conocimiento.

- Dar indicaciones básicas sobre el procedimiento metodológico.

- Revisar el plan de trabajo de cada equipo.

- Realizar reuniones con cada equipo para discutir y orientar sobre el avance del proyecto.

- Utilizar clases para satisfacer las necesidades de los equipos: repasar el temario de la asignatura y aclarar conceptos.

- Revisión individual o grupal de los progresos del proyecto y de los aprendizajes desarrollados.

- Realizar la evaluación final en base a los resultados presentados en el portafolio digital.

Cuadro 1. Funciones del profesorado en el proceso de la investigación formativa 
El alumnado construye nuevos conocimientos y habilidades trabajando desde los conocimientos y habilidades que ya posee de las asignaturas de metodología de investigación de primer y segundo curso. Deberá aprender tanto de forma autónoma como colaborativa (con su grupo de trabajo).

Las tareas del estudiante, básicamente, son:

- Conformar los grupos de trabajo.

- Interactuar con el profesor para aclarar dudas y definir el proyecto.

- Individualmente buscar y recoger información, proponer diseño y soluciones.

- Revisión de la información y planificación del trabajo.

- Desarrollo del proyecto y reuniones con el profesor.

- Entrega de un primer informe o propuesta de resultados (análisis descriptivo de la información); y un segundo informe (análisis inferencial).

- Presentación de los resultados obtenidos y de los aprendizajes logrados por el equipo en un portafolios digital y en sesiones expositivas.

Cuadro 2. Funciones del alumnado en el proceso de la investigación formativa

\section{FINALIDAD Y OBJETIVOS DEL ESTUDIO}

El estudio presenta los resultados parciales del proyecto de innovación mencionado anteriormente: El valor de la investigación formativa para la innovación y el desarrollo competencial en la educación superior, en cuanto al desarrollo de la investigación formativa mediante el aprendizaje orientado a proyectos, desde la perspectiva del alumnado participante.

Los objetivos parciales (ligados a la asignatura de Informática aplicada a la investigación educativa), fueron conocer la visión y opinión del alumnado sobre la adquisición de las competencias y la experiencia de aprendizaje mediante el aprendizaje orientado a proyectos y el portafolio digital. Se parte de la premisa fundamental que mediante la investigación formativa, se inspira al estudiantado para ahondar en su aprendizaje, reconstruirlo, darle significado, reflexionar de forma más compleja acerca del mismo, y comunicarlo de una manera más creativa.

\section{MÉTODO}

Para dar respuesta a los objetivos planteados se apostó por una metodología de encuesta, método adecuado cuando se trata de conocer la perspectiva de las personas con un enfoque cuantitativo (Torrado, 2004). Para ello, se diseñó un instrumento de medida basado en ítems escalares con cinco dimensiones, tal y como se resume en la tabla 3. 


\section{DIMENSIONES}

Datos sociodemográficos

Competencias transversales

Estrategias de enseñanza y aprendizaje

Método orientado al trabajo de investigación

Rol del profesorado

Satisfacción del alumnado

\section{ÍTEMS}

Sexo, edad, vía de acceso, dedicación a la asignatura, créditos matriculados, situación laboral, actividades de voluntariado

9 ítems de escala Likert 7 puntos

7 ítems de escala Likert 7 puntos

4 ítems de escala Likert 7 puntos

4 ítems de escala Likert 7 puntos

3 ítems de escala Likert 7 puntos

Tabla 3. Especificaciones del instrumento de medida (elaboración propia)

La alta fiabilidad de las diferentes dimensiones mediante el Alpha de Cronbach permite suponer una buena consistencia interna de sus ítems, como se puede apreciar en la tabla número 4 .

\begin{tabular}{lcc}
\multicolumn{1}{c}{ Dimensión } & Media & Alpha de Cronbach \\
\hline D1: Competencias transversales & 4,930 & 0,796 \\
\hline D2: Estrategias de enseñanza/aprendizaje & 4,974 & 0,782 \\
\hline D3: Método orientado al trabajo de investigación & 5,00 & 0,891 \\
\hline D4: Rol del profesorado & 5,525 & 0,886 \\
\hline D5: Satisfacción del alumnado & 5,312 & 0,763 \\
\hline Total & 5,031 & 0,939
\end{tabular}

Tabla 4. Especificaciones de la fiabilidad de las dimensiones (elaboración propia)

La población objeto de estudio fue el alumnado de la asignatura de Informática aplicada en la investigación educativa, que permite un máximo de 40 alumnos matriculados. Sobre esta población se aplicó la experiencia metodológica basada en ABP y portafolios digital, las dos metodologías en las que se materializó la investigación formativa. La muestra definitiva la formaron 31 estudiantes, que contestaron el cuestionario.

\section{RESULTADOS}

\section{Perfil socioacadémico de la muestra}

Mayoritariamente el alumnado matriculado en la asignatura es mujer, con una edad media de 21,14 años, manifiesta tener empleo (73\%) y la gran mayoría no participa en otras actividades de voluntariado o asociacionismo (61\%), sólo el 7\% dedica más de 10 horas a este tipo de actividades.

Tal como corresponde a los datos generales, la gran mayoría del alumnado proviene del bachillerato (77\%) y un 20\% provienen de Ciclos Formativos de Grado Superior. El 84\% del alumnado tenía matriculados 30 créditos, lo que supone una carga académica importante. 
Las percepciones del alumnado respecto a la dedicación a la asignatura son variadas. Mientras que la gran mayoría afirman que dedican menos de 5 horas semanales a la asignatura (71\%), el $58 \%$ perciben que la asignatura les ha exigido mayor dedicación que el resto de materias. En cambio, el 29\% afirma haberse dedicado de forma similar a otras asignaturas. Se avanza la hipótesis que el portafolio digital puede haber sido la causa de esta percepción de aumento de la dedicación a la asignatura.

\section{Competencias transversales}

El alumnado ha manifestado que el trabajo de investigación propuesto en la asignatura y el portafolios digital le han permitido el desarrollo de buena parte de las competencias transversales promovidas por la metodología docente, destacando en gran medida el trabajo cooperativo que se ha generado, y los procesos reflexivos, tal como puede observarse en la tabla 5.

\section{EL TRABAJO DE INVESTIGACIÓN Y EL PORTAFOLIOS EN ESTA ASIGNATURA CONSIDERO QUE ME HA PERMITIDO:}

Ser más autónomo/a

Tener más autocrítica

Ser más reflexivo/va

Ver puntos de vista diferentes al mío

Trabajar colaborativamente

Organizarme mejor el tiempo

Planificar mejor el trabajo

Tener más creatividad

Ser más consciente y expresar la vivencia del proceso de aprendizaje

TOTAL DE LA DIMENSIÓN

\section{Media}

4,84

4,97

$\mathbf{5 , 0 0}$

5,32

$\mathbf{6 , 0 3}$

4,77

4,61

4,23

4,77

44,37
Desviación típica

1,21

1,20

0,92

1,38

0,98

1,45

1,33

1,15

1,52

6,95

Tabla 5. Medias y desviaciones de los ítems sobre competencias transversales (elaboración propia)

Otras competencias que el alumnado también considera que se han reforzado con la innovación han sido la capacidad de autonomía, la visión de puntos vista diferentes al propio y la capacidad de autocrítica.

La experiencia de desarrollar un trabajo de investigación con la metodología aplicada suele ser gratificante para la gran mayoría independientemente del tiempo dedicado, ya que permite el desarrollo de varias competencias transversales vinculadas a la investigación formativa.

\section{Estrategias de enseñanza y aprendizaje para el desarrollo de competencias}

Para el desarrollo de las competencias de la asignatura, el alumnado señala la utilidad de una diversidad de estrategias de enseñanza y aprendizaje, tal como se resume en la tabla 6. Con cierta dispersión y variabilidad de opiniones, destaca de nuevo, el aprendizaje cooperativo, entendido 
como el trabajo en grupo para desarrollar el proyecto de investigación; también destaca el aprendizaje orientado a proyectos en cuanto al diseño y desarrollo del proyecto de investigación; y finalmente, el alumnado destaca las actividades de aprendizaje autónomo (estudio y trabajo individual) y de reflexión. Las tutorías es el aspecto que se señala en menor medida como útil para el desarrollo de las competencias de la asignatura. Podría ser que el rol orientador del profesorado en la misma aula minimice la importancia de las tutorías individuales.

\section{PARA ALCANZAR LAS COMPETENCIAS DE LA ASIGNATURA, EN QUÉ MEDIDA TE HAN RESULTADO ÚTILES:}

Las clases magistrales o teóricas

Las actividades de aprendizaje autónomo (estudio y trabajo individual)

El aprendizaje orientado a proyectos (el diseño y el desarrollo de un proyecto de investigación)

El aprendizaje colaborativo (el trabajo en grupo para desarrollar el proyecto anterior)

Las actividades de reflexión (portafolio digital)

El aprendizaje a través de la interacción y actividad grupal (análisis de casos y / o

la resolución de problemas prácticos en grupo).

Las tutorías

TOTAL DE LA DIMENSIÓN

\section{Media}

4,84

5,23

5,39

5,58

4,71

4,84

4,19

34,81
Desviación

típica

1,34

1,18

1,09

1,26

1,13

1,49

1,62

5,84

Tabla 6. Medias y desviaciones de los ítems sobre estrategias de enseñanza y aprendizaje (elaboración propia)

Destacamos igualmente, y con cierta curiosidad, que el alumnado ha valorado de forma similar las clases magistrales o teóricas y el aprendizaje a través de la interacción y actividad grupal para adquirir las competencias de la asignatura.

\section{Método orientado al trabajo de investigación}

Los estudiantes han señalado positivamente todos los elementos que favorecen el método orientado al trabajo de investigación. En la tabla 7 puede apreciarse que en opinión del alumnado, este método permite en gran medida buscar, usar, analizar y sintetizar la información para un aprendizaje integrador no fragmentado ni global.

Según el plan de estudios del grado de Pedagogía de la Universidad de Barcelona, esta asignatura optativa recoge los aprendizajes que el alumnado ha alcanzado en dos asignaturas metodológicas anteriores: Estadística aplicada a la educación y Teoría y práctica de la investigación educativa, siendo lógica esta dimensión de síntesis y de aprendizaje integrador que tiene la orientación de la propia asignatura. Estos datos, refuerzan la idoneidad de este método para aquellas asignaturas que integran aprendizajes globales de cada área. 


\begin{tabular}{|c|c|c|}
\hline $\begin{array}{l}\text { VALORA EN QUÉ MEDIDA CONSIDERAS QUE EL MÉTODO } \\
\text { ORIENTADO AL TRABAJO DE INVESTIGACIÓN PERMITE: }\end{array}$ & Media & $\begin{array}{l}\text { Desviación } \\
\text { típica }\end{array}$ \\
\hline Aprender a aprender & 4,97 & 1,20 \\
\hline Aprender significativamente & 4,97 & 1,05 \\
\hline $\begin{array}{l}\text { Buscar, usar, analizar sintetizar la información para un aprendizaje integrador (no } \\
\text { fragmentado, global) }\end{array}$ & 5,26 & 0,96 \\
\hline $\begin{array}{l}\text { Desarrollar un juicio crítico, implicarme más activamente en la transformación } \\
\text { social }\end{array}$ & 4,81 & 1,30 \\
\hline TOTAL DE LA DIMENSIÓN & 20,00 & 3,94 \\
\hline
\end{tabular}

Tabla 7. Medias y desviaciones de los ítems sobre el método orientado al trabajo de investigación (elaboración propia)

\section{El rol del profesorado}

En cuanto al rol del profesorado, el alumnado identifica en gran medida las diferentes características que se le plantean. Destaca, salvando la heterogeneidad de las respuestas, el rol evaluador del aprendizaje (tabla 8). Esta característica es lógica en el profesorado que utiliza una metodología docente no directiva, como es el caso del ABP en el que el estudiante aprende por descubrimiento.

\section{EN QUÉ MEDIDA LOS SIGUIENTES ASPECTOS CARACTERIZAN AL PROFESORADO DE LA ASIGNATURA:}

Proveedor / a de información

Facilitador / a de aprendizaje (mejora personal y / o académica)

Guía experto para el aprendizaje (ayuda a aprender a aprender)

Evaluador / a del aprendizaje

TOTAL DE LA DIMENSIÓN

\section{Media}

5,43

5,43

5,27

$\mathbf{5 , 9 7}$

22,10
Desviación

típica

1,57

1,50

1,53

1,13

4,99

Tabla 8. Medias y desviaciones de los ítems sobre el rol del profesorado (elaboración propia)

Destacamos también que esta dimensión ha sido la que ha obtenido una mayor puntuación, evidenciando que el alumnado ha constatado el rol no directivo del profesorado en este tipo de método.

\section{Satisfacción del alumnado}

Las puntuaciones que el alumnado señala denotan una alta satisfacción con las cuestiones planteadas. En la tabla 9 pueden consultarse las medias obtenidas, destacando la satisfacción del alumnado con la autonomía en el desarrollo de su aprendizaje. Estos resultados parecen indicar que la innovación docente implementada permite potenciar mayores niveles de autonomía en el aprendizaje del alumnado, en total coherencia con una apuesta formativa universitaria profesionalizadora y con los lineamientos del EEES. 


\begin{tabular}{|l|c|c|}
\hline DESPUÉS DE LA ASIGNATURA ME SIENTO SATISFECHO/A DE: & Media & $\begin{array}{c}\text { Desviación } \\
\text { típica }\end{array}$ \\
\hline La manera de impartir las clases & 5,16 & 1,39 \\
El proceso y el progreso de mi aprendizaje & 5,35 & 1,05 \\
La autonomía para desarrollar mi aprendizaje & $\mathbf{5 , 4 2}$ & 0,96 \\
\hline TOTAL DE LA DIMENSIÓN & 15,94 & 2,84 \\
\hline
\end{tabular}

Tabla 9. Medias y desviaciones de los ítems sobre la satisfacción del alumnado (elaboración propia)

\section{CONCLUSIONESY DISCUSIÓN}

El alumnado de la asignatura de Informática aplicada en la investigación educativa afirma que tuvo que buscar, usar, analizar y sintetizar la información para un aprendizaje integrador. En este sentido, el proyecto de investigación (como concreción del método ABP) ha favorecido el desarrollo de estas habilidades y además ha desarrollado las habilidades de investigación ya que, los alumnos han de averiguar y comprender qué es lo que pasa en torno a un tema.

Por otro lado, destacamos que las innovaciones aplicadas en la asignatura han permitido, según el alumnado, el desarrollo sobre todo de dos competencias consideradas importantes para el futuro desempeño profesional del estudiante (Blanco, 2009), como son el trabajo colaborativo y la capacidad de reflexión.

El trabajo colaborativo presenta una serie de beneficios académicos, sociales y psicológicos para el alumnado (Panitz, 2004; Benito y Cruz, 2005) a la vez que promueve habilidades y destrezas interpersonales (desempeño de roles, resolución de conflictos, responsabilidad, o expresión oral entre otras). El método por proyectos ha supuesto una oportunidad para romper el individualismo y fomentar un trabajo en colaboración en busca de soluciones comunes a la problemática planteada en la investigación, participando todos los miembros en la elaboración del producto.

La reflexión por su parte, constituye un importante recurso que favorece el compromiso del alumnado con su aprendizaje y está en la base del autoperfeccionamiento, refiriéndose al análisis realizado sobre un problema, objeto, hecho o fenómeno de la realidad (Domínguez y Gámez, 2010). El portafolio digital ha contribuido a generar procesos de reflexión, en la línea de otras experiencias con portafolios electrónicos (Rubio y Galván, 2013). Los procesos reflexivos ocupan un rol importante en la construcción de aquéllos, pues sin reflexión no hay portafolio, sino una carpeta de tareas. La reflexión contribuye en el estudiante al desarrollo de la conciencia metacognitiva y a la construcción de sus conocimientos (Sigal, 2007).

Para el desarrollo de las competencias, han resultado especialmente útiles, según el alumnado, las actividades de aprendizaje autónomo, las actividades colaborativas, las del proyecto de investigación y el portafolio. Una opinión que refuerza el valor de la propuesta docente para el desarrollo de competencias transversales. En menor medida el alumnado ha valorado también y de forma similar las clases magistrales o teóricas y el aprendizaje a través de la interacción y actividad grupal para adquirir las competencias de la asignatura. Esta misma valoración también se 
ha constatado en otros estudios (Rubio, Berlanga y Ruíz, 2013). Parece que en la representación de utilidad del alumnado convergen tanto las actividades de tipo aplicado como las que facilitan la adquisición de conocimientos. En la asignatura de Informática aplicada en la investigación educativa las escasas sesiones magistrales han sido apreciadas tal vez por el complemento que han supuesto a una metodología docente no directiva.

En conjunto el alumnado destaca haber alcanzado un nivel importante de autonomía para desarrollar el aprendizaje, siendo el aspecto con el que se siente más satisfecho tras cursar la asignatura. La autonomía permite al estudiante saber cuál es su objetivo de aprendizaje, planificando su trabajo para conseguirlo, aplicando conscientemente estrategias de aprendizaje y evaluando constantemente tanto el proceso en sí mismo como el resultado de sus prácticas de aprendizaje (Sinclair et al. 2000). En la asignatura de Informática los estudiantes han buscado y seleccionado información, han tomado decisiones sobre diferentes alternativas relacionadas con las fases de la investigación y la creación del portafolio y han valorado las consecuencias de cada elección por sí mismos.

Este resultado relacionado con la autonomía evidencia la metodología docente no directiva aplicada en la asignatura, en la que el estudiante aprende por descubrimiento, y donde el rol del profesor se orienta a evaluar, a ofrecer feed back, y a guiar el proceso de aprendizaje, tal y como también han destacado los estudiantes.

Aunque de forma especial el alumnado ha constatado un rol evaluador, en la línea de otras investigaciones con ABP donde los estudiantes manifestaron esperar del docente el reconocimiento de la tarea de aprendizaje y trabajo bien realizada (Thomas, 2000). Probablemente esta representación se ha visto reforzada por la evaluación mediante portafolio digital.

Por todo ello consideremos el valor que tienen las estrategias docentes más instrumentalizadas desde la investigación formativa en el desarrollo competencial del alumnado. Concretamente, alentar el aprendizaje a través de proyectos está demostrando efectos positivos en el fomento de la investigación, en la medida que el alumnado se involucra en un proceso dinámico e interactivo de aprendizaje. Y si bien el método de ABP en sí mismo no es novedoso y su uso se ha generalizado en los últimos años, sí que lo es en combinación con un portafolio digital y bajo el enfoque de la investigación formativa. De esta forma aumenta su potencial al unir las competencias que promueve el ABP con las que promueven los portafolios digitales, de forma que la capacidad de colaboración, reflexión y autonomía se unen para contribuir mejor a la generación de conocimientos.

\section{REFERENCIAS}

Aguaded, J. I.; López, E. y Jaén, A. (2013). Portafolios electrónicos universitarios para una nueva metodología de enseñanza superior. Desarrollo de un material educativo multimedia. Revista de Universidad y Sociedad del Conocimiento (RUSC), 10(1), 7-28. Disponible en: http:// rusc.uoc.edu/ojs/index.php/rusc/article/view/v10n1-aguaded-lopez-jaen/v10n1-aguadedlopez-jaen-es

Anzola, O. L. (2005). La investigación Formativa en los procesos de investigación asumidos por la Universidad. Reflexiones. 68-73. 
Arnau, J. (1996). Mètodes, dissenys, i tècniques en investigació psicológica. Barcelona: Edicions de la Universitat Oberta de Catalunya.

Barrett, H. (2010). Balancing the Two Faces of ePortafolios. Disponible en: http://electronicportafolios.com/systems/paradigms.html.

Benito, A. y Cruz, A. (2005). Nuevas claves para la docencia universitaria en el Espacio Europeo de Educación Superior. Madrid: Narcea.

Biggs, J., Kember, D. and Leung, D. (2001). The revised two-factor Study Process Questionnaire: R-SPQ-2F. British Journal of Educational Psychology, 71, 133-149. http://dx.doi. org/10.1348/000709901158433

Blanco, A. (2009). Desarrollo y evaluación de competencias en Educación Superior. Madrid: Narcea.

Blank, W. (1997). Authentic instruction. In W. E. Blank \& S. Harwell (Eds.), Promising practices for connecting high school to the real world (pp. 15-21). Tampa, FL: University of South Florida.

Bunge, M. (1983). La investigación cientifica. Su estrategia y filosofía. Barcelona: Ariel.

Cambridge, D. (2010). Eportfolios for Lifelong Learning and Assessment. San Francisco: JosseyBass.

Castaño, A. X. (2014). The application of eportfolio in higher education: implications on students' learning. Disponible en: http:/www.tdx.cat/bitstream/handle/10803/283262/Tesis.pdf;jsessi onid=0A6A8785722CBA1DFCC89C9EA35A07C7.tdx2?sequence $=1$

Cortés-Nieto, J.; Londoño-Toro, B.; Luna-de Aliaga, B. E.; Palacios-Sanabria, M. T. y Torres-Villareal, M. L. (2008). Investigación Formativa y nuevas propuestas pedagógicas en las Facultades de Derecho. Caso: Facultad de Jurisprudencia de la Universidad del Rosario. Studiositas, 3(1), 28-33.

De Miguel. M. (Coord.) (2006). Metodologías de enseñanza y aprendizaje para el desarrollo de competencias. Orientaciones para el profesorado universitario ante el espacio europeo de educación superior. Madrid: Alianza Editorial.

Del Rincón, D.; La Torre, A.; Arnal, J y Sans, A. (1995). Técnicas de investigación en ciencias sociales. Madrid: Dykinson.

Domínguez, M. P. y Gámez, E. (2010). La reflexión en el proceso de formación de los profesionales de la educación. Cuadernos de Educación y Desarrollo, (2) 22. Disponible en: http://www. eumed.net/rev/ced/22/pdgr.htm

Elliot, J. (1994). La Investigación-Acción en Educación. Madrid: Morata.

Guerrero, M.E. (2007). Formación de habilidades para la investigación desde el pregrado. Acta Colombiana de Psicología 2007; 10(2), 190-192.

Harwell, S. (1997). Project-based learning. In W. E. Blank \& S. Harwell (Eds.), Promising practices for connecting high school to the real world (pp. 23-28). Tampa, FL: University of South Florida.

Hernández, C. A. (2003). Investigación e investigación formativa. Nómadas, 18, 183-193.

Johnson, B. (1993). Teacher as Researcher. In: ERIC Digest.

Lyons, N. (comp.) (1999). El uso del portafolios. Propuestas para un nuevo profesionalismo docente. Argentina, Amorrortu Editores. 
Mateo, J. (2006). Claves para el diseño de un nuevo marco conceptual para la medición y evaluación educativas. Revista de Investigación Educativa, 24 (1), 165-186.

Miyahira, J. M. (2009). La investigación formativa y la formación para la investigación en el pregrado. Revista Medica Herediana, 20(3), 119-122.

Moursund, D. (1999). Project-Based Learning: Using Information Technology. Society for Technology in Education.

Neidhardt, F. C. (1997). Tradition: Research and Undergraduate Education. Report to the Regents of the University of Michigan. Disponible en: www.research.umich.edu

Panitz, T. (2004). The case for student centered instruction via collaborative learning paradigms. Disponible en: http:/www.eric.ed.gov/ERICDocs/data/ericdocs2sq1/content_ storage_01/0000019b/80/16/bd/40.pdf

Parra, C. (2004). Apuntes sobre la investigación formativa. Educación y Educadores. 7, 57-78.

Pérez Boullosa, A. (2006). Tutorías. En De Miguel, M. (Coord.). Metodologías de enseñanza y aprendizaje para el desarrollo de competencias (pp. 133-167). Madrid: Alianza Editorial.

Pérez Pérez, I. (2013). Innovación Docente en Animación Sociocultural: un modelo basado en los principios del Espacio Europeo de Educación Superior. Innovación educativa, 23, 197-213. Disponible en: http://www.usc.es/revistas/index.php/ie/article/view/964/1507.

Restrepo, G. B (2003). Conceptos y aplicaciones de la Investigación Formativa y criterios para evaluar la investigación cientifica en sentido estricto. Bogotá: CNA.

Rodríguez Illera, J. L. y Escofet, A. (2009). A learner-centered approach with the student as the producer of digital materials for hybrid courses. International Journal of Education and Development using ICT, 5 (1).

Rodríguez Illera, J. L.; Galván, C. y Martínez, F. (2013). El portafolio digital como herramienta para el desarrollo de competencias transversales. Educación y Cultura en la Sociedad de la Información, 14(2) 157- 177.

Rodríguez Sandoval, E.; Vargas, E. M. y Luna, J. (2010). Evaluación de la estrategia “aprendizaje basado en proyectos". Educación y educadores, 13(1), 13-25. Disponible en:

http://actualidadpedagogica.com/estudios_abp/\#sthash.BOxGKBe9.dpuf

Rubio, M. J. y Galván, C. (2013). Portafolios digitales para el desarrollo de competencias transversales. Aportaciones principales de los estudios con Carpeta Digital en el marco del grupo de investigación Ensenyament i Aprenentatge Virtual. Digital Education Review, 24.

Rubio, M. J.; Berlanga, V. y Ruíz, A. (2013). Identificación de actividades básicas en la adquisición de competencias profesionales de formación metodológica desde la visión del alumnado. $X V I$ Congreso internacional de Investigación e Innovación Educativa al Servicio de Instituciones y Comunidades Globales, Plurales y Diversas. Alicante: AIDIPE.

Sabariego, M. (2012). La recerca formativa. Omado (Objectes i materials docents) [en línea] [Consulta: 4 de diciembre de 2013). Disponible en: http://hdl.handle.net/2445/32423

Sabariego, M.; Ruiz, A. y Sánchez A. (2013). El valor de la investigación formativa para la innovación y el desarrollo competencial en la educación superior. En T. Ramiro-Sánchez y $\mathrm{M}^{\mathrm{a}}$ T. Ramiro. X Foro internacional sobre la evaluación de la calidad de la investigación y de la educación superior (FECIES). Granada: Asociación Española de Psicología Conductual (AEPC). 
Sabirón, F. y Arraiz, A. (2013). Aprendiendo de la evaluación: decálogo para la evaluación auténtica de competencias profesionales a través del Portafolio. Revista Iberoamericana de Evaluación Educativa, 6(1), 135-152.

Sabirón, F. y Arraiz, A. (2012). El desarrollo y evaluación de cuatro competencias complejas a través del e-portfolio en las asignaturas vinculadas al área MIDE. XVI Congreso internacional de AIDIPE, Alicante.

Saby, J. (2012). El rol del docente-investigador en el marco de la investigación formativa. PAPELES, 4(8), 23-30.

Sigal, C. (2007). El portafolio, instrumento de evaluación para promover la reflexión. XV Jornadas de Reflexión Académica en Diseño y Comunicación: "Experiencias y Propuestas en la Construcción del Estilo Pedagógico en Diseño y Comunicación”. Buenos Aires.

Sinclair, B. (2000). Learner Autonomy: the next phase? En B. Sinclair; I. McGrath y T. Lamb (Eds.). Learner autonomy, teacher autonomy: Future directions. London: Longman.

Soria, V.; Sabariego, M. y Donoso, T. (2013). El método de aprendizaje orientado a proyectos: una vía para operativizar la investigación formativa en la educación superior. En T. RamiroSánchez y $\mathrm{M}^{\mathrm{a}} \mathrm{T}$. Ramiro. X Foro internacional sobre la evaluación de la calidad de la investigación y de la educación superior (FECIES). Granada: Asociación Española de Psicología Conductual (AEPC).

South, C. (2001). Electronic Portfolios: Assessment for an advanced society. Disponible en: http:// www.techlearning.com/db_area/archives/WCE/csouth.htm

Stefani, L.; Mason, R. and Pegler, C. (2007). The Educational Potential of e-portfolios: supporting personal development and reflective learning. Oxon: Routledge.

Thomas, J. W. (2000). A Review of Research on Project-Based Learning. San Rafael (California): The Autodesk Foundation.

Tippelt, R. y Lindemann, H. (2001). El método de proyectos. Disponible en: http://www.halinco.de/ html/doces/Met-proy-APREMAT092001.pdf

Todorova, A.; Arati, D. and Osburg, T. (2010). Integrating ePortfolio in an Online Platform for Teacher Professional Development: Design and Expectations. The ePortfolio Conference at Learning Forum. London.

Torrado, M. (2004). Estudio por encuesta. En Bisquerra, R. (Coord.) Metodología de la investigación educativa (pp. 231-257). Barcelona: La Muralla.

Vilà, R.; Rubio, M. J. y Torrado, M. (2013). El uso del portafolio digital para la mejora de competencias transversales en las asignaturas metodológicas del grado de Pedagogía de la Universidad de Barcelona. I Congreso Internacional de Ciencias de la Educación y del Desarrollo. Santander: Fundación General UGR Empresa, 8-11 Octubre. Disponible en: http://www.ugr. es/ aepc/WEBEDUCACION/LIBRORESUMENESEDUCACION.pdf

Villa, A. y Poblete, M. (2007). Aprendizaje basado en competencias. Bilbao: Ediciones Mensajero.

Wiggins, G. (1990). The case for authentic assessment. Practical Assessment, Research \& Evaluation, 2(2), 2-22

Willard, K. and Duffrin, M. W. (2003). Utilizing project-based learning and competition to develop student skills and interest in producing quality food items. Journal of Food Science Education, 2, 69-73. http://dx.doi.org/10.1111/j.1541-4329.2003.tb00031.x

Zubizarreta, J. (2009). The learning portfolio. San Francisco: Anker. 